\title{
Just-in-time simulation-based training
}

\author{
Rajesh Aggarwal ${ }^{1,2}$
}

${ }^{1}$ Department of Surgery, Thomas Jefferson University, Philadelphia, Pennsylvania, USA ${ }^{2}$ Office of Strategic Business Development and Partnerships, Jefferson Health, Philadelphia, Pennsylvania, USA

\section{Correspondence to}

Dr Rajesh Aggarwal, Department of Surgery, Thomas Jefferson University, Philadelphia, Pennsylvania 19107, USA; rajesh.aggarwal@jefferson.edu

Accepted 3 August 2017

\section{SLinked}

- http://dx.doi.org/10.1136/ bmjqs-2017-006656

- http://dx.doi.org/10.1136/ bmjqs-2017-007120

CrossMark

To cite: Aggarwal R. BMJ Qual Saf 2017;26:866-868.
Simulation-based training and assessment in healthcare are now commonplace in the majority of industrialised nations. The role of standardised patients, high-fidelity and low-fidelity manikins, synthetic, animal and virtual reality platforms, and simulation suites, are accepted, and integrated into training curricula in medical and nursing schools, and residency programmes. Despite this widespread use, only a handful of studies have assessed the impact of simulation-based education on patient and health system outcomes, and these studies have their focus on procedural skills such as central line insertion or laparoscopic surgery. ${ }^{12}$ Furthermore, the emphasis of such studies has been on simulation-based education as a tool to impact early learners, with minimal consideration of its use for independent practitioners such as attending physicians and experienced nurses.

An emerging area of simulation-based education is just-in-time training, or as it was termed by Niles et al in 2009, 'rolling refreshers', which comprised a portable manikin/defibrillator system to provide automated corrective feedback to optimise cardiopulmonary resuscitation (CPR) skills of paediatric intensive care unit staff. ${ }^{3}$ All of the 420 participants were independent practitioners, including nurses, physicians and respiratory therapists, with providers who undertook frequent refresher sessions (denoted as more than two per month), achieving CPR skills success three times more rapidly than those who were infrequent users of the simulation sessions. In 2013, Scholtz et al described a study for a central venous catheter (CVC) dressing change programme, incorporating a $20 \mathrm{~min}$ simulation on a 'CVC dress rehearsal cart', delivered to 524 inpatient nurses in a tertiary care children's hospital setting. ${ }^{4}$ Nursing knowledge and self-confidence improved, there was a reduction by over half in terms of corrective prompts to complete the dressing change on patients, and most importantly, the overall central line associated blood stream infection (CLABSI) rates were reduced by $45 \%$. The authors of this study made clear that the results should not be overstated, in that there may have been confounding factors related to the decrease in CLABSI rates, such as patient and disease factors. Further studies have considered the role of just-in-time training for infant lumbar puncture, neonatal intubation and congenital cardiac surgery. ${ }^{5-7}$ Cheng et al have further described the CPR 'rolling refreshers' programme across a 10-site network of paediatric institutions, including 324 CPR-certified healthcare professionals, enrolled in a prospective randomised-controlled trial (RCT). ${ }^{8}$

In this issue of BMJ Quality \& Safety, Branzetti et al provide a valuable addition to this growing literature on justin-time training-in this case to address procedural skill decay in board-certified emergency medicine physicians. ${ }^{9}$ The authors point out that just-in-time training has been used in the manufacturing industry for rarely performed tasks and in environments that are intolerant of error. The analogy in healthcare would be a high-risk procedure that is infrequently performed, under critical time constraints, and is of direct and considerable consequence to patient outcome. The just-in-time strategy must use a specific task/intervention, which can immediately address the knowledge or skills deficit, be brief, focused and drive best practice, ideally through automated feedback.

The investigators recruited 27 emergency medicine physicians from a single institution to test their hypothesis in 
the setting of emergent transvenous pacemaker (TVP) placement, in a prospective RCT design. The authors are to be commended on the rigour of their scientific study, first with respect to content of the intervention through engagement of eight subject matter experts and appreciation of design requirements for mobile learning environments, and second with regard to the RCT itself, in terms of randomisation, primary and secondary outcome measures, power analysis, statistical tests and submission of the Consolidated Standards of Reporting Trials (CONSORT) diagram. While commonplace in many clinically based RCTs, this level of scientific underpinning is unfortunately not seen in all simulation-based research studies. ${ }^{1}$

The 25 physicians who completed all parts of the study first underwent a pretest in a simulated emergency department (ED) resuscitation bay, comprising a central line manikin for TVP placement, a trained actor as the ED nurse, and a simulation technician in a control room to control the patient's physiological parameters. The primary outcome metric of performance was a 26-point technical skill checklist, which was rated post hoc through video review, by two trained observers, with secondary measures defined as number of critical omissions, a global rating scale of performance and time taken to task completion. Subjects were randomised to just-in-time training (comprising a $30 \mathrm{~s}$ refresher video, followed by a step-by-step interactive checklist for TVP placement) or control (ie, no training), and then undertook a post-test in the exact manner as the pretest. Those in the intervention group scored significantly higher (mean 23.44 and SD 1.44) than the control group subjects (11.99 and 3.36, respectively) on the 26-point checklist, as well as on the Global Rating Scale, and made fewer critical omissions.

While a proof-of-concept study, Branzetti et al are to be congratulated on adding to the scientific literature with regard to just-in-time simulation-based training at one level, but at a broader level, to enable the healthcare community acknowledge the growing importance of simulation as a tool to improve quality and safety of clinical care, for all providers. Next steps are, of course, to translate the outcome measures to the patient and health system levels, though the infrequency of TVP procedures makes this a tough challenge. It is critical for future work in this arena to continue to blur the boundaries between simulation in stand-alone centres and the clinical setting, to ensure that it is trainees who are learners and the entire healthcare team, enshrined in the concept of lifelong learning.

Returning to the study by Branzetti et al, one aspect that could have been improved and would be advantageous for other such studies to address concerns the interaction between clinician and patient. It would have certainly been feasible to develop a hybrid simulation for TVP in this setting, through incorporation of a standardised patient lying beside the central line manikin, who was primed to behave as appropriate through the intervention. ${ }^{10}$ It may also be interesting to provide a further challenge to the clinician undertaking the TVP placement, such that the standardised patient could ask at an opportune moment why the physician was using the interactive checklist, as a challenge to their competence at performing the task. Finally, and I have mentioned this previously, there remains a focus of simulation to teach procedural skills. I can see just-in-time training to be an excellent adjunct to communication, situation awareness and leadership skills, such as the role of breaking bad news, especially if it is in a setting where the occurrence is rare, such as in an ambulatory care centre or a rehabilitation facility.

We have reached a tipping point in the role of simulation to impact healthcare safety, quality, outcomes and value-based care delivery, through integration of deliberate learning opportunities into our clinical practice, which may only take a few minutes, though could be tremendously impactful for our sickest and most fragile patients.

Competing interests RA is a consultant for Applied Medical.

Provenance and peer review Commissioned; internally peer reviewed.

(C) Article author(s) (or their employer(s) unless otherwise stated in the text of the article) 2017. All rights reserved. No commercial use is permitted unless otherwise expressly granted.

\section{REFERENCES}

1 Cook DA, Hatala R, Brydges R, et al. Technology-enhanced simulation for health professions education: a systematic review and meta-analysis. JAMA 2011;306:978-88.

2 Huang GC, McSparron JI, Balk EM, et al. Procedural instruction in invasive bedside procedures: a systematic review and meta-analysis of effective teaching approaches. BMJ Qual Saf 2016;25:281-94.

3 Niles D, Sutton RM, Donoghue A, et al. 'Rolling Refreshers': a novel approach to maintain CPR psychomotor skill competence. Resuscitation 2009;80:909-12.

4 Scholtz AK, Monachino AM, Nishisaki A, et al. Central venous catheter dress rehearsals: translating simulation training to patient care and outcomes. Simul Healthc 2013;8:341-9.

5 Kessler D, Pusic M, Chang TP, et al. Impact of just-in-time and just-in-place simulation on intern success with infant lumbar puncture. Pediatrics 2015;135:e1237-e1246.

6 Grgurich E, Arnemann C, Amon K, et al. Just-in-time video laryngoscopy versus direct laryngoscopy for neonatal intubation. J Perinat Neonatal Nurs 2016;30:367-71.

7 Olivieri LJ, Su L, Hynes CF, et al. 'Just-in-time' simulation training using 3-D printed cardiac models after congenital cardiac surgery. World J Pediatr Congenit Heart Surg 2016;7:164-8.

8 Cheng A, Brown LL, Duff JP, et al. International Network for Simulation-Based Pediatric Innovation, Research, \& Education 


\section{Editorial}

(INSPIRE) CPR Investigators. Improving cardiopulmonary resuscitation with a CPR feedback device and refresher simulations (CPR CARES Study): a randomized clinical trial. JAMA Pediatr 2015;169:137-44.

9 Branzetti JB, Adedipe AA, Gittinger MJ, et al. Randomised controlled trial to assess the effect of a Just-in-Time training on procedural performance: a proof-of-concept study to address procedural skill decay. BMJ Qual Saf 2017;26:881-91.

10 Kneebone R, Nestel D, Wetzel C, et al. The human face of simulation: patient-focused simulation training. Acad Med 2006;81:919-24. 